\title{
Optical response and excitons in gapped graphene
}

\section{Pedersen, Thomas Garm; Jauho, Antti-Pekka; Pedersen, K.}

\section{Published in:}

Physical Review B. Condensed Matter and Materials Physics

Link to article, DOI:

10.1103/PhysRevB.79.113406

Publication date:

2009

Document Version

Publisher's PDF, also known as Version of record

Link back to DTU Orbit

\section{Citation (APA):}

Pedersen, T. G., Jauho, A-P., \& Pedersen, K. (2009). Optical response and excitons in gapped graphene. Physical Review B. Condensed Matter and Materials Physics, 79(11), 113406.

https://doi.org/10.1103/PhysRevB.79.113406

\section{General rights}

Copyright and moral rights for the publications made accessible in the public portal are retained by the authors and/or other copyright owners and it is a condition of accessing publications that users recognise and abide by the legal requirements associated with these rights.

- Users may download and print one copy of any publication from the public portal for the purpose of private study or research.

- You may not further distribute the material or use it for any profit-making activity or commercial gain

- You may freely distribute the URL identifying the publication in the public portal

If you believe that this document breaches copyright please contact us providing details, and we will remove access to the work immediately and investigate your claim 


\title{
Optical response and excitons in gapped graphene
}

\author{
Thomas G. Pedersen, ${ }^{1}$ Antti-Pekka Jauho, ${ }^{2,3}$ and Kjeld Pedersen ${ }^{1}$ \\ ${ }^{1}$ Department of Physics and Nanotechnology, Aalborg University, DK-9220 Aalborg Øst, Denmark \\ ${ }^{2}$ Department of Micro and Nanotechnology, DTU Nanotech, Technical University of Denmark, DK-2800 Kongens Lyngby, Denmark \\ ${ }^{3}$ Laboratory of Physics, Helsinki University of Technology, P.O. Box 1100, 02015 HUT, Finland
}

(Received 23 September 2008; revised manuscript received 3 February 2009; published 20 March 2009)

\begin{abstract}
Graphene can be rendered semiconducting via energy gaps introduced in a variety of ways, e.g., coupling to substrates, electrical biasing, or nanostructuring. To describe and compare different realizations of gapped graphene we propose a simple two-band model in which a "mass" term is responsible for the gap. The optical conductivity predicted for this model is obtained as a simple closed-form expression. In addition, analytical estimates for the binding energy of excitons are derived and the impact of excitons on the optical response is analyzed.
\end{abstract}

DOI: 10.1103/PhysRevB.79.113406

PACS number(s): 78.67.-n, 71.35.Cc

\section{INTRODUCTION}

Graphene has an enormous potential for ultrasmall scale electronic applications ${ }^{1-3}$ due to its structural stability and huge mobility reaching values up to $200000 \mathrm{~cm}^{2} / \mathrm{V} \mathrm{s}^{3,4}$ The lack of a band gap in the celebrated Dirac energy spectrum of ideal graphene, however, is a severe restriction for many applications. Fortunately, a substantial band gap can be induced in several ways. Graphene grown epitaxially on $\mathrm{SiC}$ has a band gap of about $0.2 \mathrm{eV}$ due to interaction with the substrate. ${ }^{5}$ The presence of the substrate invariably leads to a substantial electron doping, however. Biasing a graphene bilayer also leads to the formation of a gap, ${ }^{6}$ but applying large voltages across such minute distances is challenging. An alternative route to large and controllable energy gaps is via structuring at the nanoscale. For instance, sculpturing graphene into nanoribbons produces a gap that can be varied via the width and orientation of the ribbon. ${ }^{7}$ Also, large-area samples of graphene will develop a gap if a periodic array of circular holes (antidot lattice) is introduced. ${ }^{8,9} \mathrm{We}$ have previously analyzed this option and find a gap that scales favorably with geometry. ${ }^{8}$ Hence, energy gaps up to $0.5 \mathrm{eV}$ can be produced in this manner. The realization of such large-area gapped graphene samples could enable fabrication of a wide range of electronic and optoelectronic devices. Recently, sculpturing of roughly circular holes with $3.5 \mathrm{~nm}$ diameter in few-layer graphene was demonstrated, ${ }^{10}$ indicating that graphene antidot lattices are indeed realistic structures.

The optical response of gapped graphene is of importance for several reasons. First, it is needed for an understanding of optoelectronic devices such as photodetectors and lightemitting devices. Second, optical spectroscopy might be applied for measurements of the magnitude of the energy gap. In Ref. 9, a comprehensive study of the optical response of graphene antidot lattices was presented. It was demonstrated that these structures are direct-gap semiconductors and that clear spectroscopic signatures appear at the band edge. In the present work, we wish to generalize this work in two directions. Primarily, a more "universal" description of the response of gapped graphene is desirable. By "universal" we mean a model that includes an adjustable energy gap but ignores the detailed manner in which this gap is produced (substrate, electrical bias, nanoscale sculpturing, etc.). This will highlight the role of the magnitude of the gap and enable simple comparison between different realizations of gapped graphene. Second, excitons are expected to modify the optical response significantly. This is because one expects a strong electron-hole interaction in graphene: the twodimensional character leads to increased overlap between electron and hole wave functions, and the screening is in general weak, both in suspended graphene and graphene placed on dielectric substrates. To meet these goals, we develop in the present work a simplified model of the electronic structure of gapped graphene, in which the gap is produced by adding a constant "mass" term to the usual twoband graphene model. By nature, a constant mass term should be a better description of structures that preserve their two-dimensional character (graphene on substrates, antidot lattices, etc.) than quasi-one-dimensional structures such as nanoribbons, for which the model will be less accurate. However, this model is sufficiently simple that an analytic expression for the optical conductivity can be found. Moreover, in the vicinity of the band gap, this model reduces to an isotropic effective-mass description with an exceedingly simple value for the reduced effective electron-hole pair mass. Hence, excitonic effects can be estimated using a twodimensional effective-mass picture. To illustrate our findings, we compare the optical response for different values of the energy gap. Furthermore, the substantial influence of excitonic effects on the spectra is demonstrated and implications for detection of the band edge are discussed.

\section{GAPPED GRAPHENE}

In a nearest-neighbor tight-binding model using $\pi$-electron states on the two sublattices as basis functions, the electronic states are found as eigenfunctions of the Hamiltonian, ${ }^{11}$

$$
H=\left(\begin{array}{cc}
0 & -\gamma f^{*}(\vec{k}) \\
-\gamma f(\vec{k}) & 0
\end{array}\right),
$$

where $\gamma=3.033 \mathrm{eV}$ (Ref. 11) is the transfer integral and $f$ is given by 


$$
f(\vec{k})=e^{i k_{x} a / \sqrt{3}}+2 e^{-i k_{x} a / 2 \sqrt{3}} \cos \left(k_{y} a / 2\right) .
$$

The energy eigenvalues are $E= \pm \gamma|f(\vec{k})|$ and the two bands touch at the $K$ point $\vec{K}=(\sqrt{3}, 1) \cdot 2 \pi / 3 a$, where $a$ is the lattice constant, leading to a vanishing energy gap of regular graphene. Linearization of the model around $\vec{k}=\vec{K}$ yields the celebrated description of carriers as massless Dirac fermions. $^{2}$ In this description, mass can be reintroduced by the simple generalization,

$$
H=\left(\begin{array}{cc}
\alpha & -\gamma f^{*}(\vec{k}) \\
-\gamma f(\vec{k}) & -\alpha
\end{array}\right),
$$

which results in energies $E= \pm \sqrt{\alpha^{2}+\gamma^{2}|f(\vec{k})|^{2}}$ and correspondingly a gap of $E_{g}=2 \alpha$ at the $K$ point. Formally, this model also arises in a tight-binding model of graphene with on-site potentials $\alpha$ and $-\alpha$ for the two sublattices, respectively, or in the description of a boron nitride layer in which the two $\mathrm{C}$ atoms of the graphene unit cell are replaced by a BN dimer. ${ }^{12}$ In addition, it can be seen as an approximate model of Dirac fermions in an antidot lattice, provided the spatially varying "mass" term is replaced by an effective average value. ${ }^{8}$ In all cases, the model Eq. (3) can be viewed as an effective description provided the appropriate value of $\alpha$ is adopted. It is clear, however, that the present model is only a simplistic approximation for the actual structures. Hence, the predicted results are expected to be valid only in cases where the gap is produced by a minor perturbation and only for an energy range in the vicinity of the gap. Below, results for antidot lattices are used to illustrate this fact.

Apart from its universal nature, the great value of the present model is its mathematical similarity to that of regular graphene. The close similarity implies that several known analytical results for regular graphene can be adapted to the gapped case provided appropriate adjustments are made. For the optical response, the starting point is the transition energy $E_{c v}=2 \sqrt{\alpha^{2}+\gamma^{2}|f(\vec{k})|^{2}}$. For regular graphene, the expression reduces to $\varepsilon_{c v}=2 \gamma|f(\vec{k})|$. Thus, gapped and regular cases are related via $E_{c v}^{2}=\varepsilon_{c v}^{2}+E_{g}^{2}$. To illustrate the importance of this relation, we first consider the joint density of states $D(\omega)$,

$$
D(\omega)=\int \delta\left(E_{c v}-\hbar \omega\right) d^{2} k
$$

Using well-known scaling properties of delta functions, it follows that

$$
\begin{aligned}
D(\omega) & =2 \hbar \omega \int \delta\left(E_{c v}^{2}-\hbar^{2} \omega^{2}\right) d^{2} k \\
& =2 \hbar \omega \int \delta\left(\varepsilon_{c v}^{2}+E_{g}^{2}-\hbar^{2} \omega^{2}\right) d^{2} k \\
& \equiv 2 \hbar \omega \int \delta\left(\varepsilon_{c v}^{2}-\hbar^{2} \widetilde{\omega}^{2}\right) d^{2} k=\frac{\omega}{\widetilde{\omega}} \int \delta\left(\varepsilon_{c v}-\hbar \widetilde{\omega}\right) d^{2} k
\end{aligned}
$$

The integral is recognized as the joint density of states of regular graphene but with a shifted argument $\widetilde{\omega} \equiv \sqrt{\omega^{2}-\omega_{g}^{2}}$, where $\omega_{g}=E_{g} / \hbar$. Hence, the results of Ref. 13 immediately imply that

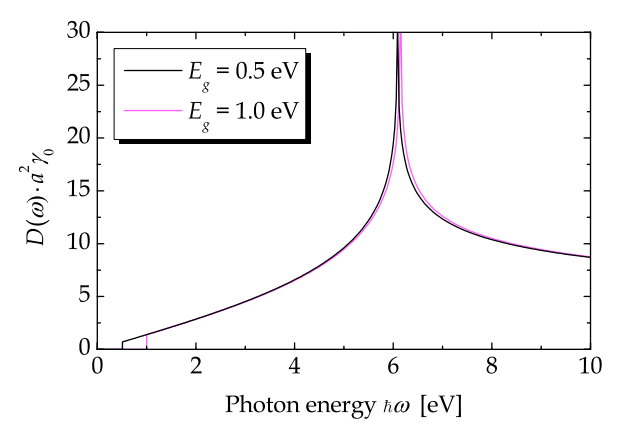

FIG. 1. (Color online) Joint density of states for two characteristic values of the energy gap.

$D(\omega)=\left(4 \Omega / a^{2} \gamma \sqrt{6 \widetilde{\Omega}}\right) \operatorname{Re}\left\{K\left[(6-\widetilde{\Omega})(2+\widetilde{\Omega})^{3} / 128 \widetilde{\Omega}\right]\right\}$,

where $K$ is the complete elliptic integral of the first kind and we have introduced normalized frequencies $\Omega=\hbar \omega / \gamma$ and $\widetilde{\Omega}=\hbar \tilde{\omega} / \gamma$. Also, it is understood that $D(\omega)=0$ for $\omega<\omega_{g}$ and for $\tilde{\Omega}>6$. This simple result is illustrated in Fig. 1 for two values of the energy gap. It is clear that, apart from the forbidden gap, the joint density of states is only weakly affected by the presence of an energy gap. This is, of course, a consequence of the fact that the band structure rapidly approaches that of regular graphene for energies above or below the gap.

The optical conductivity $\widetilde{\sigma}(\omega)$ is more complicated than the joint density of states as the $k$-dependent momentum matrix element must be taken into account. Effects due to disorder and imperfections can conveniently be modeled by a phenomenological broadening parameter, as we shall discuss below. Here, we first consider the ideal case, when the real part $\sigma(\omega)$ is given by the formula

$$
\sigma(\omega)=\left(e^{2} / 2 \pi m^{2} \omega\right) \int\left|P_{v c}\right|^{2} \delta\left(E_{c v}-\hbar \omega\right) d^{2} k,
$$

where $P_{v c}$ is the in-plane momentum matrix element, and $m$ is the free-electron mass. The somewhat lengthy derivation of $P_{v c}$ can be carried out along the lines of Ref. 13 and when summed over the two in-plane directions, the result can be written as,

$\left|P_{v c}\right|^{2}=\left(\varepsilon_{c v}^{2} / E_{c v}^{2}\right)\left|P_{v c}^{0}\right|^{2}-(m a / \hbar)^{2}\left(E_{g}^{2} / 6 E_{c v}^{2}\right)\left(\varepsilon_{c v}^{2} / 4\right)-9 \gamma^{2}$,

where $\left|P_{v c}^{0}\right|^{2}$ is the value of regular graphene. A simple calculation using the scaling procedure described above then demonstrates that

$$
\begin{aligned}
\sigma(\omega)= & \left(\widetilde{\omega}^{2} / \omega^{2}\right) \sigma^{0}(\widetilde{\omega})-\left(\omega_{g}^{2} / \omega^{3}\right)\left[\left(\widetilde{\omega}^{2} / 4\right)-\left(9 \gamma^{2} / \hbar^{2}\right)\right] \\
& \times\left(e^{2} a^{2} / 24 \pi\right) D(\omega),
\end{aligned}
$$

where $\sigma^{0}(\omega)$ is the conductivity of regular graphene and $D(\omega)$ is the joint density of states found above. Inserting Eq. (6) and utilizing our previous derivation of $\sigma^{0}(\omega),{ }^{9,13}$ we finally find

$$
\begin{aligned}
\sigma(\omega) & =\left(\sigma_{0} / \pi \Omega^{2}\right) \sqrt{(2 \widetilde{\Omega} / 3)} \\
& \times \operatorname{Re}\left\{\left(144 \widetilde{\Omega}-84 \widetilde{\Omega}^{2}+3 \widetilde{\Omega}^{4}+72 \Omega^{2}-2 \Omega^{2} \widetilde{\Omega}^{2} / 24 \widetilde{\Omega}\right)\right. \\
& \times K\left[(6-\tilde{\Omega})(2+\widetilde{\Omega})^{3} / 128 \widetilde{\Omega}\right]
\end{aligned}
$$




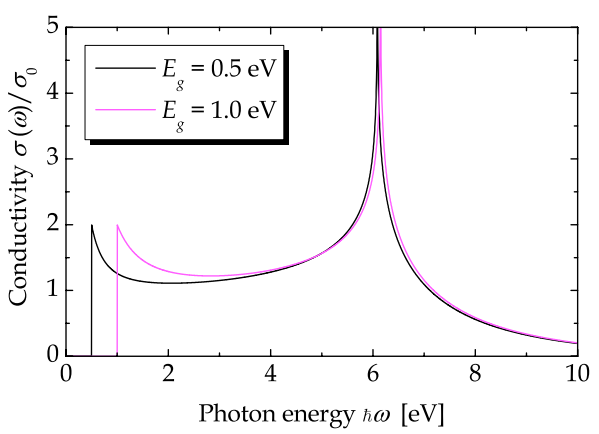

FIG. 2. (Color online) Analytical conductivity spectra for two examples of gapped graphene.

$$
\left.-12 E\left[(6-\widetilde{\Omega})(2+\widetilde{\Omega})^{3} / 128 \widetilde{\Omega}\right]\right\} .
$$

Here, $E$ is the complete elliptic integral of the second kind and $\sigma_{0}=e^{2} / 4 \hbar$ is the dc value of graphene. This expression reduces to the graphene case if the limit $\widetilde{\Omega} \rightarrow \Omega$ is taken. Also, it can be demonstrated that $\sigma\left(\omega_{g}\right)=2 \sigma_{0}$ and that slightly above the gap $\sigma(\omega) \approx 2 \sigma_{0} \omega_{g}^{2} / \omega^{2}$ in agreement with the corresponding derivation for gapped graphene in the linearized Dirac model. ${ }^{14}$ The conductivity is plotted for some representative cases in Fig. 2.

We have carefully checked the correctness of the analytic expression Eq. (10) by comparison to a direct numerical evaluation of Eq. (7) using the improved triangle method. ${ }^{9}$ Also, to validate the gapped graphene model, we have compared the optical spectra to the numerical results for graphene antidot lattices. ${ }^{9}$ In this particular realization, energy gaps are introduced by forming an array of circular holes in a graphene sheet and the magnitude of the gap depends strongly on the hole diameter. In Fig. 3, we have compared numerical antidot lattice spectra to the predictions of the present model using energy gaps adjusted to fit the antidot values. It is noted that the gapped graphene spectra are quite reasonable approximations, especially for the lower band-gap case. The two cases represent different degrees of perturbation of the ideal graphene structure and, as expected, larger deviations are observed for a larger perturbation. Obviously, far above the band gap, the numerical antidot spectra deviate substantially from the gapped graphene model as a consequence of the complicated antidot lattice band structure. However, in the vicinity of the band edge the gapped

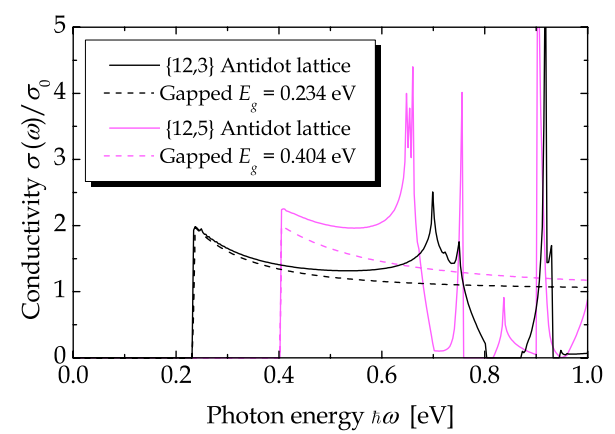

FIG. 3. (Color online) Comparison of spectra for graphene antidot lattices and gapped graphene. The energy gaps have been adjusted to fit two particular antidot lattice geometries.

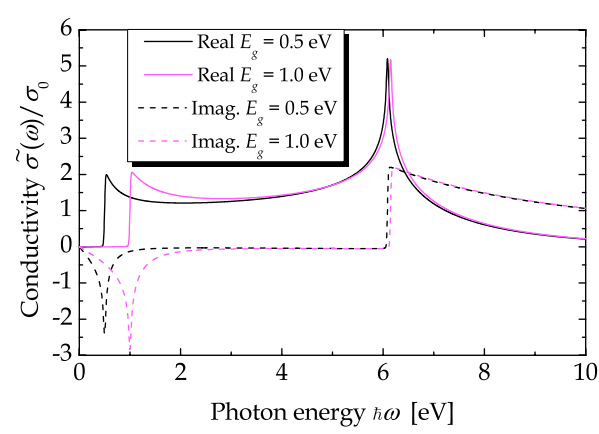

FIG. 4. (Color online) Complex conductivity of gapped graphene assuming a broadening of $50 \mathrm{meV}$.

graphene model is a reasonable approximation. At this point, broadening can be introduced and the imaginary part of the complex conductivity computed. To this end, we first convolute the real part of the conductivity with a Gaussian broadening of $50 \mathrm{meV}$ and subsequently apply the KramersKronig transformation. The resulting spectra are shown in Fig. 4.

\section{EXCITONS IN GAPPED GRAPHENE}

As gapped graphene is a low-dimensional semiconductor and screening in most situations is expected to be weak, excitonic effects will severely modify the optical response, especially below the band gap. The influence of excitons on the optical response of graphene nanoribbons has previously been studied using a highly accurate but computationally expensive Bethe-Salpeter approach. ${ }^{15,16}$ The predicted exciton binding energies close to $1 \mathrm{eV}$ testify strongly to the significance of excitons in gapped graphene generally. In the Wannier model, the binding energy of excitons is determined by the effective Rydberg energy Ry* of a particular realization of gapped graphene. To provide a quantitative estimate we note that the transition energy $E_{c v}$ expanded around the $K$ point can be written as

$$
E_{c v} \approx E_{g}+\left(3 \gamma^{2} a^{2} / 4 \alpha\right) k^{2}=E_{g}+\left(2 \hbar^{2} v_{F}^{2} / E_{g}\right) k^{2},
$$

where $v_{F}=\sqrt{3} \gamma a / 2 \hbar$ is the Fermi velocity. Hence, the dispersion is that of an isotropic parabola and writing the result in the form of an effective-mass dispersion $E_{c v}=E_{g}+\hbar^{2} k^{2} / 2 \mu$, we find a reduced effective electron-hole pair mass of $\mu$ $=E_{g} / 4 v_{F}^{2}$. Thus, the effective mass is simply proportional to the band gap, similarly to the case of carbon nanotubes. ${ }^{17}$ To evaluate $\mathrm{Ry}^{*}$ we also need the appropriate dielectric constant $\varepsilon$ screening the Coulomb interaction. This in itself is a complicated quantity depending on the details of the sample and its surroundings. As an illustrative example, we will consider a sample positioned on the surface of a $\mathrm{SiO}_{2}$ substrate and, for simplicity, assume that screening by the substrate is the dominating mechanism. In this case, the resulting dielectric constant can simply be taken as the average between that of the substrate $\varepsilon_{\mathrm{SiO}_{2}}$ and that of air leading to $\varepsilon=\left(\varepsilon_{\mathrm{SiO}_{2}}+1\right) / 2$ $\approx 2.5{ }^{18}$ Since $v_{F} \approx 10^{6} \mathrm{~m} / \mathrm{s}$, the effective mass for a band gap of 0.5 and $1.0 \mathrm{eV}$ becomes $0.022 \mathrm{~m}$ and $0.044 \mathrm{~m}$, respectively. In turn, the effective Rydbergs $\mathrm{Ry}^{*}=13.6 \mathrm{eV} \mu / m \varepsilon^{2}$ for these cases are 48 and $96 \mathrm{meV}$, respectively. These large 
values indicate that, indeed, excitons are of importance in gapped graphene. We stress, however, that these values of $\mathrm{Ry}^{*}$ are only the relevant energy scale for extended Wannier excitons with vanishing screening by the gapped graphene itself.

We will continue to work within the effective-mass approximation to estimate qualitatively the influence on the full optical response spectrum. In this approximation, the binding energy of the lowest $(1 s)$ exciton equals $4 \mathrm{Ry}^{*}$, which is clearly a substantial fraction (around 38\%) of the band gap. Assuming, in addition, $k$-independent momentum matrix elements $p_{v c}$ yields the two-dimensional (2D) Elliott formula ${ }^{19}$ for the excitonic conductivity $\sigma_{X}(\omega)$,

$$
\begin{gathered}
\sigma_{X}(\omega)=\sigma_{\mathrm{FC}}(\omega)\left\{\sum_{n=0}^{\infty} \frac{4 \delta\left(\Delta+\left(n+\frac{1}{2}\right)^{-2}\right)}{\left(n+\frac{1}{2}\right)^{3}}\right. \\
\left.+\frac{2 \theta(\Delta)}{1+\exp (-2 \pi / \sqrt{\Delta})}\right\},
\end{gathered}
$$

where $\Delta=\left(\hbar \omega-E_{g}\right) / \mathrm{Ry}^{*}, \theta$ is the unit-step function, and $\sigma_{\mathrm{FC}}(\omega)=e^{2} \mu\left|p_{v c}\right|^{2} /\left(\hbar^{2} m^{2} \omega\right)$ is the free-carrier conductivity. It follows that a simple estimate for the excitonic spectrum of gapped graphene can be obtained using Eq. (10) as the appropriate free-carrier expression. In the contribution from bound excitons we take, for simplicity, $\sigma_{\mathrm{FC}}(\omega) \approx \sigma_{0}$. Hence,

$$
\sigma_{X}(\omega) \approx \sigma_{0} \sum_{n=0}^{\infty} \frac{4 \delta\left[\Delta+\left(n+\frac{1}{2}\right)^{-2}\right]}{\left(n+\frac{1}{2}\right)^{3}}+\sigma(\omega) \frac{2 \theta(\Delta)}{1+\exp (-2 \pi / \sqrt{\Delta})} .
$$

The excitonic spectrum is illustrated in Fig. 5 for the cases discussed above, i.e., Ry* $=48 \mathrm{meV}$ and $96 \mathrm{meV}$. Here, broadening of $50 \mathrm{meV}$ is assumed and the free-carrier spectra are included for comparison. The most prominent difference is clearly the large $1 s$ resonance. The higher excitons are included in the calculations but due to their lower oscillator strength and close proximity to the band edge, they are not resolved in the spectra. If slightly larger broadening is assumed, the $1 s$ resonance will merge with the continuum, in particular in the case of low band gaps. This may make it

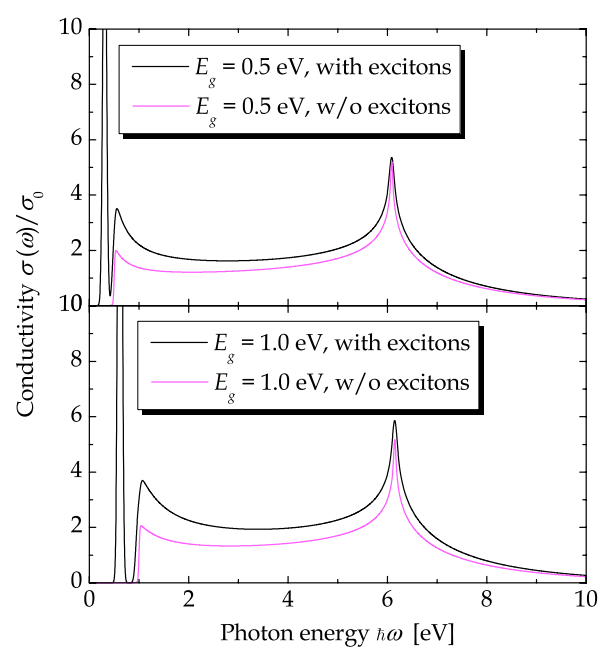

FIG. 5. (Color online) Comparison of spectra with and without excitons for gapped graphene on a $\mathrm{SiO}_{2}$ substrate. The two panels illustrate cases of medium and large band gaps.

difficult to clearly identify the band edge in an optical spectroscopic measurement. In this case, fitting the experimental spectrum with a model such as the present one may provide an estimate of the band edge.

\section{SUMMARY}

In summary, a simple two-band model of gapped graphene has been proposed as an approximate description of various physical realizations of semiconducting graphene. The gap is introduced as a spatially constant "mass" term. The simplicity of the model implies that important spectra such as the joint density of states and the optical conductivity can be obtained completely analytically. Near the band edge, the model is reduced to a simple isotropic effective-mass description with an effective-mass proportional to the energy gap. Based on these findings, the effect of excitons on the optical response is estimated. Within the Wannier model, we estimate that exciton binding energies for samples placed on $\mathrm{SiO}_{2}$ substrates are approximately $40 \%$ the energy gap.

Financial support from Danish Research Council FTP grant "Nanoengineered graphene devices" is gratefully acknowledged.
${ }^{1}$ C. Berger et al., J. Chem. Phys. B108, 19912 (2004).

${ }^{2}$ A. K. Geim and K. S. Novoselov, Nature Mater. 6, 183 (2007).

${ }^{3}$ X. Du et al., Nat. Nanotechnol. 3, 491 (2008).

${ }^{4}$ K. I. Bolotin et al., Solid State Commun. 146, 351 (2008).

${ }^{5}$ S. Y. Zhou et al., Nature Mater. 6, 770 (2007).

${ }^{6}$ E. V. Castro et al., Phys. Rev. Lett. 99, 216802 (2007).

${ }^{7}$ X. Li et al., Science 319, 1229 (2008).

${ }^{8}$ T. G. Pedersen et al., Phys. Rev. Lett. 100, 136804 (2008); Phys. Rev. Lett. 100, 189905(E) (2008).

${ }^{9}$ T. G. Pedersen et al., Phys. Rev. B 77, 245431 (2008).

${ }^{10}$ M. D. Fischbein and M. Drndić, Appl. Phys. Lett. 93, 113107
(2008).

${ }^{11}$ R. Saito et al., Physical Properties of Carbon Nanotubes (Imperial College Press, London, 1998).

${ }^{12}$ V. A. Margulis et al., Phys. Rev. B 77, 035425 (2008).

${ }^{13}$ T. G. Pedersen, Phys. Rev. B 67, 113106 (2003).

${ }^{14}$ V. N. Kotov et al., Phys. Rev. B 78, 075433 (2008).

${ }^{15}$ L. Yang et al., Nano Lett. 7, 3112 (2007).

${ }^{16}$ D. Prezzi et al., Phys. Rev. B 77, 041404(R) (2008).

${ }^{17}$ T. G. Pedersen, Phys. Rev. B 67, 073401 (2003).

${ }^{18}$ T. Ando, J. Phys. Soc. Jpn. 75, 074716 (2006).

${ }^{19}$ M. Shinada and S. Sugano, J. Phys. Soc. Jpn. 21, 1936 (1966). 